arXiv: math.PR/0000000

\title{
Supplementary section for "Bayesian Nonparametric Estimation of Milky Way Parameters Using Matrix-Variate Data, in a New Gaussian Process Based Method"
}

\author{
Dalia Chakrabarty $^{*, \S}$, Munmun Biswas ${ }^{\dagger, \uparrow}$, Sourabh Bhattacharya ${ }^{\ddagger, \Upsilon}$ \\ , \\ $\S$ Department of Statistics \\ University of Warwick \\ Coventry CV4 7AL, U.K. \\ d. chakrabarty@warwick.ac.uk \\ and \\ Department of Mathematics \\ University of Leicester \\ Leicester LE1 7RH, U.K. \\ dc252@le.ac.uk \\ I Indian Statistical Institute \\ 203, B. T. Road \\ Kolkata 700108, India \\ munmun.biswas08@gmail.com sourabh@isical.ac.in
}

Throughout, we refer to our main manuscript as CBB.

\section{Background of the Application}

As indicated in Figure 1, we approximate the geometry of the Milky Way disc as a 2-dimensional disc. Thus, we confine analysis to such a two-dimensional spatial geometry, rendering the location vector of the $a$-th star as $\left(X_{1}^{(a)}, X_{2}^{(a)}\right)^{T}$, and the velocity vector as $\left(U^{(a)}, V^{(a)}\right)^{T}$. Let such locations and velocities of $j$ stars be measured. It is to be emphasised that $\left(X_{1}^{(a)}, X_{2}^{(a)}\right)^{T}$ is the measurement taken from the Sun (i.e. from or location in he Galaxy), i.e. $\left(X_{1}^{(a)}, X_{2}^{(a)}\right)^{T}$ is the heliocentric location of the $a$-th star. Thus, if the Solar location with respect to the Galactic centre is $\left(r_{\odot}, \phi_{\odot}\right)^{T}$, then the value of the Galactocentric location of the $a$-th star is $\left(r_{\odot}, \phi_{\odot}\right)^{T}+\left(x_{1}^{(a)}, x_{2}^{(a)}\right)^{T}$. To put this in the context of the lower

\footnotetext{
*Associate Research fellow at Department of Statistics, University of Warwick and Lecturer of Statistics at Department of Mathematics, University of Leicester

${ }^{\dagger} \mathrm{PhD}$ student in Statistics and Mathematics Unit, Indian Statistical Institute

$¥$ Assistant Professor in Bayesian and Interdisciplinary Research Unit, Indian Statistical Institute
} 
panel in Figure 1, the general location vector to a star at point $C$ inside the sampled region centred at $S$, is along line-segment $O C$ and is given by the sum of the location vectors along $O S$ and $S C$. However, the Galactocentric stellar location is unknown as is the Solar location $\left(r_{\odot}, \phi_{\odot}\right)^{T}$. Thus, using the measured value $\left(x_{1}^{(a)}, x_{2}^{(a)}\right)^{T}$, the unknown $\left(r_{\odot}, \phi_{\odot}\right)^{T}$ cannot be estimated. Furthermore, the spatial locations of the sampled $j$ stars lie inside a circle with radius $\epsilon$ centred at the Sun (Fux, 2001), and are assumed to be distributed uniformly within this circle. Then the summary of the distribution of the measured locations $\left\{\left(x_{1}^{(a)}, x_{2}^{(a)}\right)^{T}\right\}_{a=1}^{j}$ will always coincide with the centre of this circle - which is the Sun - irrespective of what the galactocentric location of this centre is. Thus, these measured spatial locations cannot constrain the sought galactocentric location of the Sun.

Similarly, the recorded values of stellar velocities, $\left(u^{(a)}, v^{(a)}\right)^{T}, a=1, \ldots, j$, are as measured from the Sun and are therefore with respect to the solar velocity. These measured heliocentric stellar velocities are however affected by the choice of the location of the observer, i.e. the location of the Sun, i.e. $\left(r_{\odot}, \phi_{\odot}\right)^{T}$. This is because, a given star, if observed from different spatial locations in the Galaxy, would appear to move in different ways. For example, as indicated in Figure 1, if a star appears to have a velocity vector directed along the line that joins itself to the observer at point $A$ on the Milky Way disc, this observer will register its velocity to be entirely radial, with zero angular component of the velocity vector. Here "radial" component of the velocity vector is the component along the line-of-sight joining the observer to the star and the component orthogonal to the line-of-sight is referred to as the "angular" component. On the contrary, had the observer been at a different point $B$, the velocity vector of this star would have registered to have had a radial as well as an angular component, in general. Thus, the observed stellar velocities will bear information about the location of the observer, i.e. the Sun. Then the available velocity data $\mathbf{V}$ can be considered to bear the signature of the unknown $\boldsymbol{S}$. In principle, beyond just the galactocentric solar location, if there are Milky Way feature parameters that physically affect stellar motions, observed velocity data will bear the signature of such Milky Way parameters.

\section{Details of dynamical simulations of astrophysical models}

In Chakrabarty (2007), the simulations involve the following. A sample of stellar 2-dimensional location and 2-dimensional velocity coordinates $\left\{r^{(a)}, \phi^{(a)}, u^{(a)}, v^{(a)}\right\}_{a=1}^{j}$, is drawn from a chosen (to mimic real disc galaxies') density function $g(R, \Phi, U, V)$ at $T=0$, and is evolved in a (chosen) parametric Galactic gravitational potential $\Psi(R, \Phi, T)$ where we recall that the strength and shape of the gravitational influence of the system is given by the gravitational potential. Here $T$ is time variable. In fact, the gravitational influence of the system is modelled as mostly due to the Milky Way disk, but perturbed by the gravitational potential of the central bar in the Galaxy (see Fig 1 above) as well as that of the Galaxy's spiral arms. The potential of the disk is assumed to be stationary and chosen to emulate a 

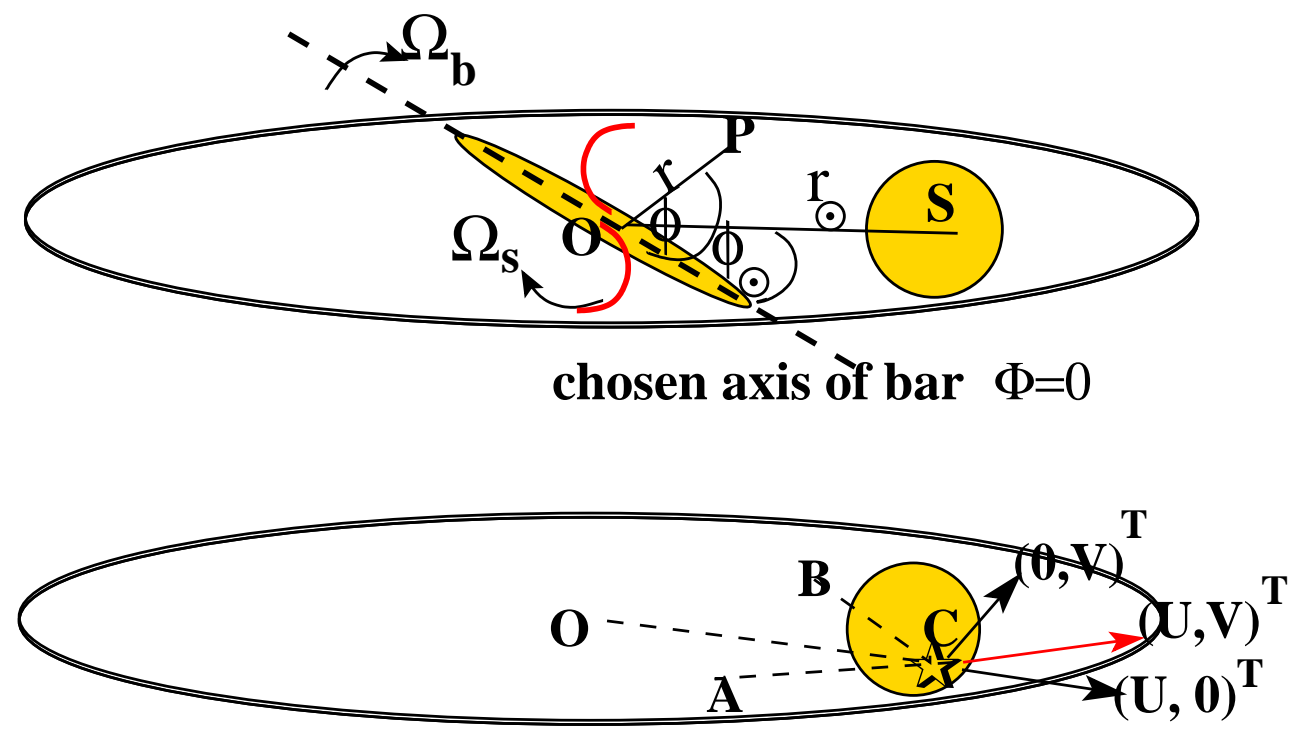

Fig 1. Top: schematic diagram of the Milky Way disk centred at point marked $O$ with the Sun at the point marked $S$. The long axis of the central rotating stellar bar (marked in broken lines) is chosen to define $\Phi=0$, where $\Phi$ is the variable that measures the angular separation of a point on the disk from this axis. The variable $R$ measures the distance of a point on the disk from the Galactic centre $O$. Thus, at a general point $P, R=r$ and $\Phi=\phi$. At the Sun at $S, R=r_{\odot}, \Phi=\phi_{\odot}$. The 2-dimensional velocity vectors of a sample of stars lying within a circle centred at $S$, are observed by us from Earth, i.e. from the Sun. The Galactic spiral arms are schematically shown in red. The bar and spiral rotate with angular speeds of $\Omega_{b}$ and $\Omega_{s}$ respectively. Bottom: one of the sampled stars (marked by the $\star$ symbol within the circle centred at the Sun) at point $C$, would appear to have velocity $(u, v)^{T}$ with respect to the Galactic centre at $O$. This means that an observer at $O$ would register a "radial" component $(u, 0)^{T}$ along her line-of-sight to the star, i.e. along the line $O C$, and a "angular" component $(0, v)^{T}$ orthogonal to the line-of-sight. However, if another observer at the point A viewed this star - where the line segment AC lies along vector $(u, v)^{T}$ - she will regard the projection of the $(u, v)^{T}$ vector onto a line orthogonal to the line segment $A C$, to be zero, i.e. will view the velocity of the star to be entirely along her line of sight. The radial component (component along line of sight) of this star's velocity according to her would be $\sqrt{u^{2}+v^{2}}$ while the angular component (orthogonal to line of sight) is 0 , so that she will observe this star to move with velocity $\left(\sqrt{u^{2}+v^{2}}, 0\right)^{T}$. Another viewer at point $B$ will however infer different values of the radial and transverse components of the velocity of this star, given the orientation of the location $B$ with respect to the vector $(u, v)^{T}$.

realistic, time-independent background Galactic potential $\Psi_{0}(R, \Phi)$. The contribution of the potential of the rotating (and therefore time-dependent) Galactic bar is $\varepsilon_{b}(T) \Psi_{b}(R, \Phi, T)$ where the scalar $\varepsilon_{b}(T)$ represents the strength of the disturbance that the bar imposes on the disk's gravitational influence at time $T$. Again, $\varepsilon_{b}(T)$ is chosen to emulate the growth of the bar inside the Galaxy. We recall that the bar is chosen to rotate about the centre of the disk at a rate of $\Omega_{b}$. Similarly, the potential of the rotating spiral pattern is $\varepsilon_{s}(T) \Psi_{s}(R, \Phi, T)$. 
Again, $\Omega_{s}$ defines the rotation rate of the spiral pattern. Thus at any time and at any location on the disk, the net gravitational potential in the $q$-th base astronomical model is $\Psi_{0}^{(q)}(R, \Phi)+\varepsilon_{b}(T) \Psi_{b}^{(q)}(R, \Phi, T)+\varepsilon_{s}(T) \Psi_{s}^{(q)}(R, \Phi, T)$, for each $q=1, \ldots, 4$. The sampled stellar location and velocity coordinates are made to evolve using Newtonian equations of motion under the influence of the aforementioned net gravitational potential. At the end of a chosen period of time, when $T=t_{\text {sim }}$, evolved orbits are sampled and recorded in the rotating frame of the bar at times when $\left(\Omega_{b}-\Omega_{s}\right) t=0$.

The relevant subset of the space of the design vectors (i.e. chosen solar location vector) is discretised and the recorded orbits are sorted by their final locations into the discretised bins; thus stars with final locations in the neighbourhood of the centroid of the $i$-th discretised bin were slotted into the $i$-th bin. The interpretation of this is that stars in the $i$-th bin share a similar galactocentric location $s_{i}^{\star}$, and their $j$ number of $k$-dimensional velocity vectors comprise the $i$-th synthetic velocity data set, (which we treat as the $j k$-dimensional vector). Here $i=1,2, \ldots, n$ and Chakrabarty (2007) used $n=216$. The $n$ synthetic velocity vectors, each thus generated at the $n$ grid points, form the training data set $\mathcal{D}_{s}$. The grid that the design vectors are grid points of is defined by the ranges of $r \in[1.7,2.3]$ in model units and $\phi \in[0,90]$ in degrees. The same 2-D grid is used for each of the base astrophysical models.

In fact, in any base astrophysical model of the Milky Way, all distances are in units of the "co-rotation radius" $r_{C R}$ of the central bar in the Milky Way disc. This is the radius at which the rotational rate $\Omega_{b}$ of the rotating bar, equals the radius-dependent rotational rate $\Omega(R)$ of the stars at distance $R$ from the centre of the Galaxy, which in turn is determined by the choice of inputs in the base astrophysical models. In all 4 of the base astrophysical models, this stellar rotational rate $\Omega(R)$ is defined as $\frac{v_{0}}{R}$, where $v_{0}$ is a constant that is set to unity in the base astrophysical models by Chakrabarty (2007); $\Omega_{b}$ is also set to unity. Then co-rotation occurs when $\Omega(R)=\Omega_{b}$, i.e. $\frac{v_{0}}{r_{C R}}=\Omega_{b}$ which implies that $r_{C R}=1$ in each of the 4 base astrophysical models.

To connect any distance in these base models to a physically realised distance measured in units of kilo parsec-or $\mathrm{kpc}-$ one needs to

- scale the constant $v_{0}$ to its real astronomical value of $220 \mathrm{kms}^{-1}$ (Binney and Merrifield, 1998) and

- scale the bar rotational rate $\Omega_{b}$ to its real astronomical value so that $r_{C R}=\frac{v_{0}}{\Omega_{b}}$ is computed in real physical units. However it is the value of $\Omega_{b}$ in astronomical units that remains elusive and is sought. So, we

- scale our estimate of the solar radial location to the Galactic centre, $\hat{r_{\odot}}$, to the distance $\mathcal{R}$ measured in $\mathrm{kpc}$, as cited in astronomical literature (obtained using ancillary information in independent astronomical modelling). This gives the scaling between model units and astronomical units (kpc) so that a distance of 1 in model units then follows as $\frac{\mathcal{R}}{\hat{r}_{\odot}} \mathrm{kpc}$, i.e. $r_{C R}$ 
Chakrabarty, Biswas and Bhattacharya/Estimation using New $\mathcal{G P}$ Based Method-Supplement

in real units is $\frac{\mathcal{R}}{\hat{r}_{\odot}} \mathrm{kpc}$. Independent astronomical studies have suggested $\mathcal{R}=8 \mathrm{kpc}$ (Binney and Merrifield, 1998). We then use this real value of $r_{C R}$ in $\frac{v_{0}}{r_{C R}}=\Omega_{b}$ to get an estimate of the sought $\Omega_{b}$. Thus, $\Omega_{b}=\frac{v_{0}}{\frac{\mathcal{R}}{\hat{r}_{\odot}}}$. Using $v_{0}=220 \mathrm{kms}^{-1}$ and $\mathcal{R}=8 \mathrm{kpc}$, we get $\Omega_{b}=\frac{220}{\frac{8}{\hat{r}_{\odot}}} \mathrm{kms}^{-1} / \mathrm{kpc}$. Learning the rotational rate $\Omega_{b}$ of the bar is the ulterior benefit of learning the solar radial location as in our approach.

\section{TMCMC algorithm}

Motivated by the fact that the performance of traditional MCMC methods - including the Metropolis-Hastings algorithm - can be less than satisfactory in high dimensions, both in terms of convergence and computational time, Dutta and Bhattacharya (2013) proposed the Transformation based MCMC or TMCMC. Dutta and Bhattacharya (2013) show that for additive transformations, the TMCMC-based acceptance rate decreases at a slower rate compared to block random walk Metropolis algorithms. Furthermore, TMCMC includes the hybrid Monte Carlo (HMC) algorithm as a special case and in one-dimensional situations while it boils down to the Metropolis-Hastings algorithm with a specialised proposal mechanism.

For our purpose, we shall consider TMCMC based on additive transformations, since Dutta and Bhattacharya (2013) show that these transformations require far less number of "move types" compared to non-additive transformations.

TMCMC allows updating the entire block $\left(\boldsymbol{s}^{(\text {new })}, \boldsymbol{Q}, \boldsymbol{\Sigma}\right)$ at the same time. The algorithm is as follows.

(i) Initialise the unknown quantities by fixing arbitrary initial values $\left(\boldsymbol{s}^{(n e w, 0)}, \boldsymbol{Q}^{(0)}, \boldsymbol{\Sigma}^{(0)}\right)$. In our case, $\boldsymbol{s}^{(n e w, 0)}=\left(s_{1}^{(n e w, 0)}, \ldots, s_{d}^{(\text {new }, 0)}\right), \boldsymbol{Q}^{(0)}$ is characterised by the initial values of the $d$ smoothness parameters, which we denote by $\boldsymbol{b}:=\left(b_{1}^{(0)}, \ldots, b_{d}^{(0)}\right)^{T}$ and $\boldsymbol{\Sigma}^{(0)}$ denotes the initial choice of the $k \times k$ matrix $\boldsymbol{\Sigma} . \boldsymbol{\Sigma}$ is decomposed into $\boldsymbol{L} \boldsymbol{L}^{T}$, where $\boldsymbol{L}$ is the appropriate lower-triangular matrix

(ii) Let $\boldsymbol{\varphi}=\left(\left(\boldsymbol{s}^{(\text {new })}\right)^{T}, \boldsymbol{b}^{T},\left(\boldsymbol{L}^{*}\right)^{T}\right)^{T}$, where $\boldsymbol{L}^{*}$ denotes the column vector consisting of the non-zero elements of $\boldsymbol{L}$.

(iii) Next we propose $\epsilon \sim g(\cdot) I_{\{\epsilon>0\}}$, where $g(\cdot)$ is some arbitrary distribution, and $I$ denotes the indicator function. In our applications, we shall choose $g(\cdot)=N(0,1)$, so that, $\epsilon>0$ is drawn from a truncated normal distribution.

(iv) Assume that at iteration $t$, the state of the unknown parameters is $\left(\boldsymbol{s}^{(n e w, t)}, \boldsymbol{Q}^{(t)}, \boldsymbol{\Sigma}^{(t)}\right):=$ $\varphi^{(t)}$. Update $\varphi^{(t)}$ by setting, with probabilities $\pi_{j}$ and $\left(1-\pi_{j}\right), \varphi_{j}^{(t+1)}=$ $\varphi_{j}^{(t)} \pm c_{j} \epsilon$ (forward transformation) and $\varphi_{j}^{(t+1)}=\varphi_{j}^{(t)}-c_{j} \epsilon$ (backward 
transformation), respectively, where, for $j=1, \ldots, d, \pi_{j}$ are appropriately chosen probabilities and $c_{j}$ are appropriately chosen scaling factors. Assume that for $j_{1} \in \mathcal{U}, \varphi_{j_{1}}^{(t)}$ gets the positive transformation, while for $j_{2} \in \mathcal{U}^{c}, \boldsymbol{\varphi}_{j_{2}}^{(t)}$ gets the backward transformation. Here $\mathcal{U} \cup \mathcal{U}^{c}=\left\{1, \ldots, d^{*}\right\}$, where $d^{*}=2 d+\frac{k(k+1)}{2}$.

(v) We accept the new proposal $\varphi^{(t+1)}$ with acceptance probability

$$
\alpha_{\varphi}=\min \left\{1, \frac{\prod_{j_{1} \in \mathcal{U}}\left(1-\pi_{j_{1}}\right) \prod_{j_{2} \in \mathcal{U}^{c}} \pi_{j_{2}}}{\prod_{j_{1} \in \mathcal{U}} \pi_{j_{1}} \prod_{j_{2} \in \mathcal{U}^{c}}\left(1-\pi_{j_{2}}\right)} \times r_{\varphi}\right\}
$$

where $r_{\boldsymbol{\varphi}}$ denotes the ratio of $\left[\left|\boldsymbol{A}_{D_{\text {aug }}}\left(\boldsymbol{s}_{\text {aug }}\right)\right|^{-\frac{j k}{2}}\left|\boldsymbol{H}_{D_{\text {aug }}}^{T}\left(\boldsymbol{s}_{\text {aug }}\right) \boldsymbol{A}_{D_{\text {aug }}}^{-1}\left(\boldsymbol{s}_{\text {aug }}\right) \boldsymbol{H}_{D_{\text {aug }}}\left(\boldsymbol{s}_{\text {aug }}\right)\right|^{-\frac{j k}{2}}\right] \times$ $\left[|\boldsymbol{\Sigma}|^{-\frac{k(n+1-m)+k+1}{2}}\left|(n+1-m) k \hat{\boldsymbol{C}}_{G L S, a u g}\right|^{-\frac{(n+1-m) k}{2}}\right]$, evaluated at the new value $\left(\varphi^{(t+1)}\right)$ and the current value $\left(\varphi^{(t)}\right)$ of $\varphi$ respectively. We only need to bear in mind that the acceptance probability is zero if $b_{j} \leq 0$ for any $j$ or if any diagonal element of $\boldsymbol{L}$ is negative.

For proper choices of the scale parameters of the additive transformation and the initial values of the parameters we conducted several initial "pilot" TMCMC runs of length around 100,000, starting with arbitrary initial values and guesses of the scale parameters such that all the runs converged to the same distribution as indicated by informal diagnostics such as trace plots. For the final TMCMC run, we chose those scale parameters that yielded the best convergence (with respect to empirical diagnostics such as trace plots) among the pilot runs, and selected the final values of the parameters obtained in this best pilot run as the initial values for the final run of TMCMC. The pilot runs yielded the proposal mechanism that we worked with.

\section{Cross-validation}

We employ leave-one-out cross-validation to assess the validity of our model and methodology. We leave out the $i$-th value $\boldsymbol{s}_{i}$ of the model parameter vector $\boldsymbol{S}$ and predict this $\boldsymbol{s}_{i}$ using the data that comprises motion vector $\mathbf{v}_{i}$, along with the remaining training data set from which $\mathbf{v}_{i}$ is omitted. Then for this prediction, the test data is really $\mathbf{v}_{i}$; to emphasise this form of the test data in notation similar to what we have used above, we denote the test data as $\mathbf{v}^{(t e s t, i)}$ where $\mathbf{v}^{(t e s t, i)}:=\mathbf{v}_{i}$. The training data set relevant to this exercise is obtained by omitting the $i$-th row from $\mathcal{D}_{s}$, i.e. the training data $\mathcal{D}_{s}^{(-i)}$ is constructed as $\mathcal{D}_{s}$ bereft of the $j k$-dimensional motion vector $\mathbf{v}_{i}$. The aim is to compute the posterior probability density of $s_{i}$, given the relevant test and training data sets. We perform such leave-one-out cross-validation for each $i=1,2, \ldots, n$. To perform inference with $\mathbf{v}_{i}$ omitted, a TMCMC run is required, implying that the full cross-validation would then demand $n$ many TMCMC runs. Such is however computationally burdensome. Bhattacharya and Haslett (2007) have shown that the usual importance sampling/resampling methods suggested by 
Gelfand, Dey and Chang (1992) and Gelfand (1996), which may be effective in the case of forward problems, are not appropriate for inverse problems because of the technical intricacies of the latter. Bhattacharya and Haslett (2007) suggested a fast methodology for implementing cross-validation in inverse problems, by combining importance resampling (IR) and low-dimensional MCMC runs in an effective manner. We adopt this methodology, which the above authors termed IRMCMC, but replace the MCMC part with the more effective TMCMC methodology.

In the following we discuss the procedure for model validation.

1. Choose an initial $i^{*}$ where $\pi\left(\boldsymbol{S}, \boldsymbol{Q}, \boldsymbol{\Sigma} \mid \mathcal{D}_{s}^{\left(-i^{*}\right)}, \mathbf{v}^{\left(\text {test }, i^{*}\right)}\right)$ as the importance sampling density. Bhattacharya and Haslett (2007) demonstrate that an appropriate $i^{*}$ may be obtained by minimising the following distance function with respect to $i$ :

$$
d(i)=\sum_{t=1}^{n}\left\{\sum_{u=1}^{d} \frac{\left(s_{t}^{(u)}-s_{i}^{(u)}\right)^{2}}{\nu_{s_{u}}^{2}}+\sum_{\ell=1}^{j k} \frac{\left(\mathbf{v}_{t}^{(\ell)}-\mathbf{v}_{i}^{\ell}\right)^{2}}{\nu_{v_{\ell}}^{2}}\right\},
$$

where $\nu_{s_{u}}^{2}$ and $\nu_{v_{u}}^{2}$ are the data-based standard deviations corresponding to the $u$-th coordinate of $s$ and $\mathbf{v}$, respectively.

2. From this importance sampling density, following Section 3, use TMCMC to sample $\left(\boldsymbol{s}^{(\ell)}, \boldsymbol{Q}^{(\ell)}, \boldsymbol{\Sigma}^{(\ell)}\right), \ell=1, \ldots, N$ for large $N$.

3. For $i \in\left\{1, \ldots, i^{*}-1, i^{*}+1, \ldots, n\right\}$,

a. for each sample value $\left(\boldsymbol{s}^{(\ell)}, \boldsymbol{Q}^{(\ell)}, \boldsymbol{\Sigma}^{(\ell)}\right)$, compute importance weights $w_{i^{*}, i}^{(\ell)}=w_{i^{*}, i}\left(\boldsymbol{s}^{(\ell)}, \boldsymbol{Q}^{(\ell)}, \boldsymbol{\Sigma}^{(\ell)}\right)$, where the importance weight function is given by

$$
w_{i^{*}, i}(\boldsymbol{s}, \boldsymbol{Q}, \boldsymbol{\Sigma})=\frac{L\left(\boldsymbol{s}, \boldsymbol{Q}, \boldsymbol{\Sigma} \mid \mathcal{D}_{s}^{(-i)}, \mathbf{v}^{(t e s t, i)}\right)}{L\left(\boldsymbol{s}, \boldsymbol{Q}, \boldsymbol{\Sigma} \mid \mathcal{D}_{s}^{\left(-i^{*}\right)}, \mathbf{v}^{\left(t e s t, i^{*}\right)}\right)}
$$

where $L\left(\boldsymbol{s}, \boldsymbol{Q}, \boldsymbol{\Sigma} \mid \mathcal{D}_{s}^{(-i)}, \mathbf{v}^{(\text {test }, i)}\right)$ is proportional to the posterior $\left[\boldsymbol{s}, \boldsymbol{Q}, \boldsymbol{\Sigma} \mid \mathcal{D}_{s}^{(-i)}, \mathbf{v}^{(t e s t, i)}\right]$ which is given in Equation 2.13 of CBB.

b. For $j \in\left\{1, \ldots, J_{1}\right\}$

(i) Sample $\left(\tilde{\boldsymbol{Q}}^{(j)}, \tilde{\boldsymbol{\Sigma}}^{(j)}\right)$ from $\left\{\left(\boldsymbol{Q}^{(1)}, \boldsymbol{\Sigma}^{(1)}\right), \ldots,\left(\boldsymbol{Q}^{(N)}, \boldsymbol{\Sigma}^{(N)}\right)\right\}$ without replacement, where the probability of sampling $\left(\boldsymbol{Q}^{(\ell)}, \boldsymbol{\Sigma}^{(\ell)}\right)$ is proportional to $w_{i^{*}, i}^{(\ell)}$.

(ii) For fixed $(\boldsymbol{Q}, \boldsymbol{\Sigma})=\left(\tilde{\boldsymbol{Q}}^{(j)}, \tilde{\boldsymbol{\Sigma}}^{(j)}\right)$, draw $\boldsymbol{s} J_{2}$ times from posterior density $\left[\boldsymbol{s} \mid \boldsymbol{Q}, \boldsymbol{\Sigma}, \mathcal{D}_{s}^{(-i)}, \mathbf{v}^{(\text {test }, i)}\right]$ using TMCMC, where for this choice of $\boldsymbol{Q}$ and $\boldsymbol{\Sigma}$,

$$
\left[\boldsymbol{S} \mid \boldsymbol{Q}, \boldsymbol{\Sigma}, \mathcal{D}_{s}^{(-i)}, \mathbf{v}^{(t e s t, i)}\right] \propto\left[\boldsymbol{S}, \boldsymbol{Q}, \boldsymbol{\Sigma} \mid \mathcal{D}_{s}^{(-i)}, \mathbf{v}^{(t e s t, i)}\right]
$$

In this way, $J_{2}$ samples of $s$ are obtained at each $J_{1}$.

c. Store the $J_{1} \times J_{2}$ draws of $s$ as the $J_{1} J_{2}$ number of posterior samples for $\boldsymbol{s}_{i}$ as $\hat{\boldsymbol{s}}_{i}^{(1)}, \ldots, \hat{\boldsymbol{s}}_{i}^{\left(J_{1} J_{2}\right)}$. 


\subsection{Simulation study}

In order to perform the cross-validation discussed above, on simulated data, we contrive a situation where there are $j=3$ stars, each having $k=2$ velocity components where velocity $\mathbf{v}=\boldsymbol{\xi}(\boldsymbol{s})=\left(\xi_{1}(\boldsymbol{s}), \ldots, \xi_{6}(\boldsymbol{s})\right)^{T}$, with the model parameter $\boldsymbol{S}$ being of dimension $d=2, s=\left(s^{(1)}, s^{(2)}\right)$ (the two coordinates of the solar position). We assign the following forms to the component functions of the 6-dimensional vector-valued function $\boldsymbol{\xi}(\cdot)$.

$$
\begin{aligned}
& \xi_{1}(s)=\alpha s^{(1)}+\beta \frac{s^{(2)}}{1+\left(s^{(1)}\right)^{2}}+\gamma \cos \left(1.2 s^{(2)}\right) \\
& \xi_{2}(s)=\alpha s^{(1)}+\beta \frac{s^{(2)}}{1+\left(s^{(1)}\right)^{2}} \\
& \xi_{3}(s)=\alpha+\beta s^{(1)} \\
& \xi_{4}(s)=\gamma \cos \left(1.2 s^{(2)}\right) \\
& \xi_{5}(s)=\alpha s^{(1)}+\gamma \cos \left(1.2 s^{(2)}\right) \\
& \xi_{6}(s)=\gamma \cos \left(s^{(2)}+\sin \left(s^{(2)}\right)\right),
\end{aligned}
$$

where $\alpha, \beta$ and $\gamma$ are chosen constants. Most of these above forms are modified versions of the functional forms used in Carlin, Polson and Stoffer (1992) and Bhattacharya (2007) in connection with dynamic models; see also Ghosh et al. (2013).

We generated 100 data points by first simulating $\boldsymbol{s}_{i}=\left(s_{i}^{(1)}, s_{i}^{(2)}\right) ; i=1, \ldots, 100$ independently from Uniform $(-1,1) \times \operatorname{Uniform}(-1,1)$, and then evaluating $\xi(\cdot)$ at each $s_{i}$, using the component-wise functional forms given above (4.3)-(4.8). Here we set $\alpha=0.05, \beta=0.1$ and $\gamma=0.2$. We thus obtained 100 data points $\left(s_{i}, \mathcal{V}_{i}\right) ; i=1, \ldots, 100$.

We leave out each data point in turn, predicting the corresponding location using the remaining data points and the corresponding velocity matrix. Using the distance minimisation method discussed in the last sub-section we obtain $i^{*}=43$; hence the importance sampling density is $\left[\boldsymbol{s}, \boldsymbol{Q}, \boldsymbol{\Sigma} \mid \mathbf{v}_{43}, \sqsubseteq^{(t e s t, i)}\right]$.

\subsection{Details of IRMCMC implementation to simulated data}

We implemented TMCMC following the details provided in Section 3.1 of CBB in order to simulate from the importance sampling density at $i^{*}=43$. Specifically, for updating $s$ using TMCMC, the parameter $\epsilon$-a scaled value of which the proposed state is an additive transformation-is chosen to be $\epsilon \sim N(0,1) I_{\{\epsilon>0\}}$ while the scale factors $c_{1}$ and $c_{2}$ are chosen to be 0.1 and 50 . For the smoothness parameters, i.e. the elements of the diagonal matrix $\boldsymbol{Q}$, we simulated $\epsilon$ from a zero mean normal distribution with variance 0.005 , restricted to $\mathbb{R}_{+}$, and selected 0.1 and 1 as the scale factors. For updating $\boldsymbol{\Sigma}$, we simulated the transformation parameter $\epsilon$ from the positively restricted zero mean normal distribution with variance 0.005 and set the scale factors to be 0.07 for the non-zero 

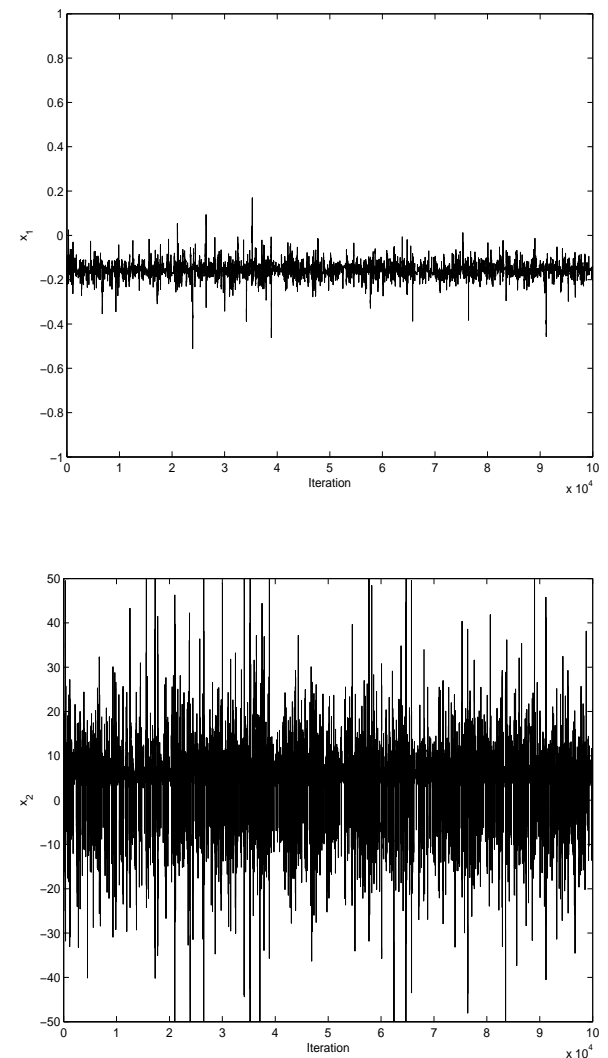

FIG 2. Simulation study: Trace plots of $s_{1}$ and $s_{2}$ corresponding to $i^{*}=43$.

elements. These choices are arrived at after assessing TMCMC convergence in several pilot runs.

We discarded the first 100,000 TMCMC runs corresponding to the obtained $i^{*}$ as burn-in and stored the next 100,000 runs for IR purposes. Informal convergence diagnostics indicated reasonable convergence; see, for example, the trace plots of $s_{1}$ and $s_{2}$ in Figure 2. From these 100,000 samples we simulated 100 realisations of $(\boldsymbol{Q}, \boldsymbol{\Sigma})$ using IR without replacement. For each IR-realised $(\boldsymbol{Q}, \boldsymbol{\Sigma})$ we simulated 1000 realisations of $s$ using TMCMC; In this implementation of TMCMC we used a burn-in of 100,000 iterations of $\boldsymbol{s}$, starting from an initial value generated uniformly over $[-1,1] \times[-1,1]$. Thereafter, for the remaining 99 IR-realisations, we used the last realisation of $s$ as the initial value for the first realisation of $s$, without discarding any iteration as burn-in. This was done at the previous IR-realisation of $(\boldsymbol{Q}, \boldsymbol{\Sigma})$. That this is a valid and efficient strategy, has been established by Bhattacharya and Haslett (2007). Thus, we obtain $100 \times 1000=100,000$ IRMCMC realisations of $s$. Each such set of 100,000 reali- 
sations was generated for each omitted data point.

This entire exercise took around 49 hours on a laptop; posterior simulation corresponding to $i^{*}$ took around one hour, while the remaining exercise took a further period of 48 hours approximately. It is to be noted that brute-force cross-validation in this example would have taken 100 hours. Hence IRMCMC lives up to the expectation of reducing the computation time.

\subsection{Results of cross-validation on simulated data}

In all of the $100 \%$ simulated cases, the true locations fell within the $95 \%$ highest posterior density credible intervals of the corresponding leave-one-out IRMCMC-based posteriors. Some of the leave-one-out cross-validation posteriors, along with the corresponding true values are shown in Figures 3 and 4 . The results indicate a good fit to the data and are therefore encouraging as far as the application of our high-dimensional Gaussian process-based method is concerned.

\subsection{IRMCMC-based cross-validation using data generated from base astrophysical models}

For each of the four base astrophysical models (bar_6, sp3bar3, sp3bar3_18 and sp3bar3_25), we have a training data set consisting of 216 observations on 100dimensional motion vectors, each generated at a distinct value of the design vector. In order to validate our Gaussian process based methodology we perform leave-one-out cross-validation for each of the four training data sets using IRMCMC in conjunction with TMCMC.

\subsection{Prior on location in the context of cross-validation}

For the cross-validation purpose, we assume a somewhat expanded parameter space for the locations: $\left(R_{\odot}, \Phi_{\odot}\right) \in(1,3) \times[0, \pi)$, instead of the parameter space $[1.7,2.3] \times[0, \pi / 2]$, which was assumed for actually predicting the unknown location associated with the real, test data set. The reason for expanding the parameter space is that the training data sets consist of many observations that lie almost on the boundary of $[1.7,2.3] \times[0, \pi / 2]$ and our initial cross-validation showed that many boundary values were excluded from the $95 \%$ credible regions of their respective cross-validation posteriors. Indeed, for both classical and Bayesian asymptotics the important regularity condition that is typically assumed is that the true value of the parameter lies within the interior of the parameter space (page 436 of Schervish (1995)).

Note that the aforementioned expansion of the parameter space of $\left(R_{\odot}, \Phi_{\odot}\right)$ for cross-validation purpose is not in conflict with the uniform prior on $[1.7,2.3] \times$ $[0, \pi / 2]$, which we assumed for predicting the unknown location corresponding to the real, training data set. Indeed, guided by the astrophysics literature we 

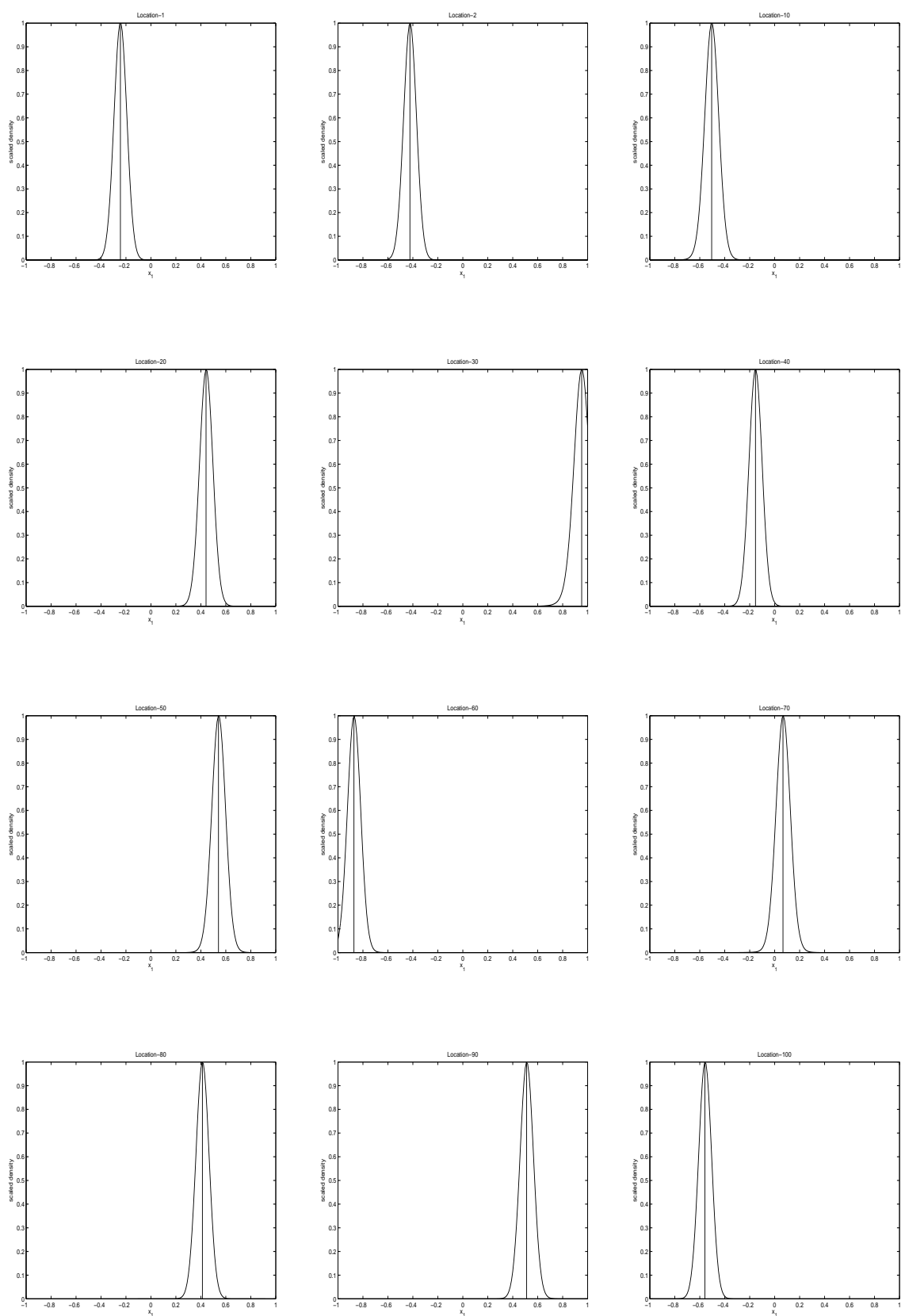

FIG 3. Simulation study: Leave-one-out cross-validation posteriors of $s_{1}$; the vertical line indicates the true value. 

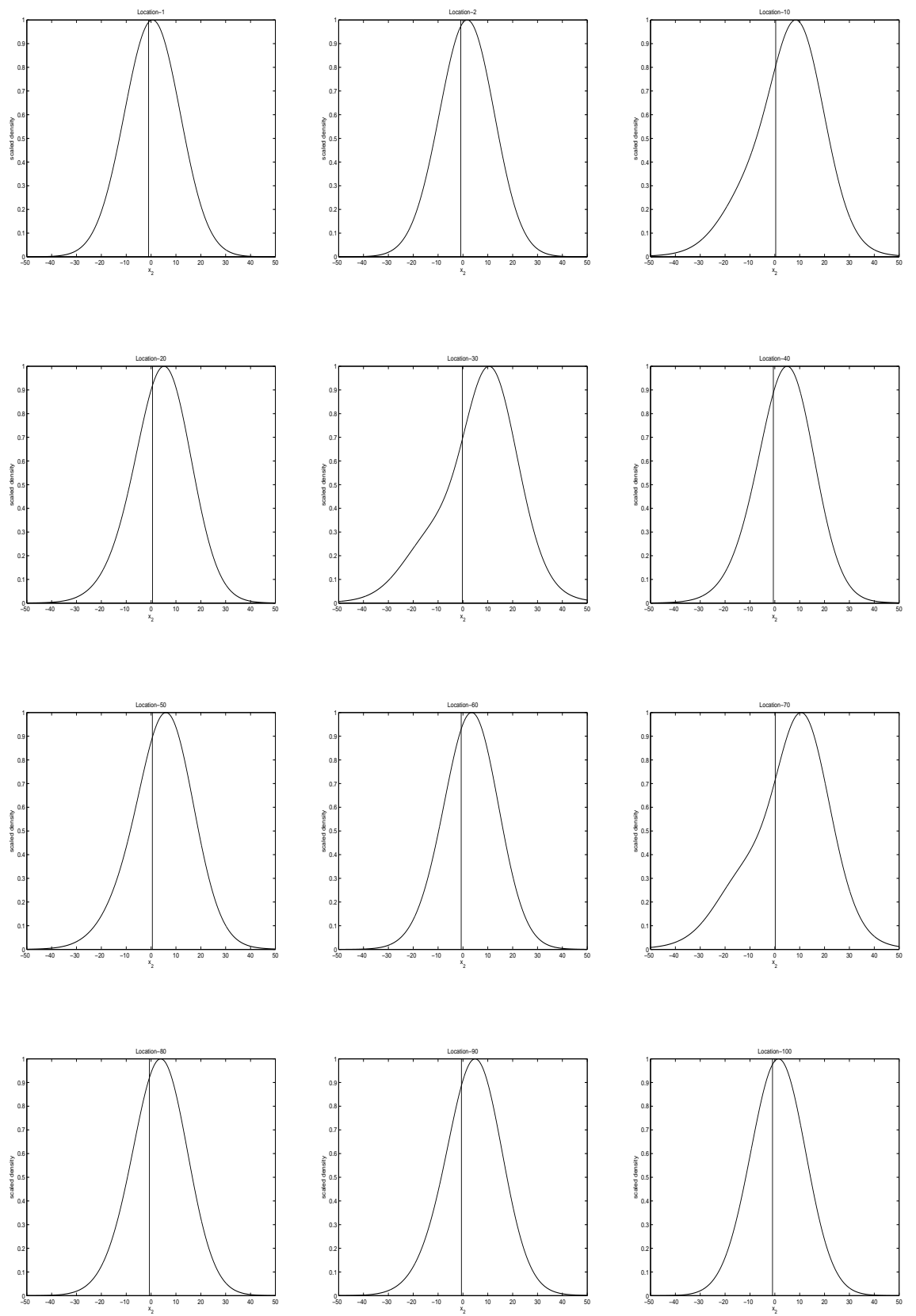

FIG 4. Simulation study: Leave-one-out cross-validation posteriors of $s_{2}$; the vertical line indicates the true value. 

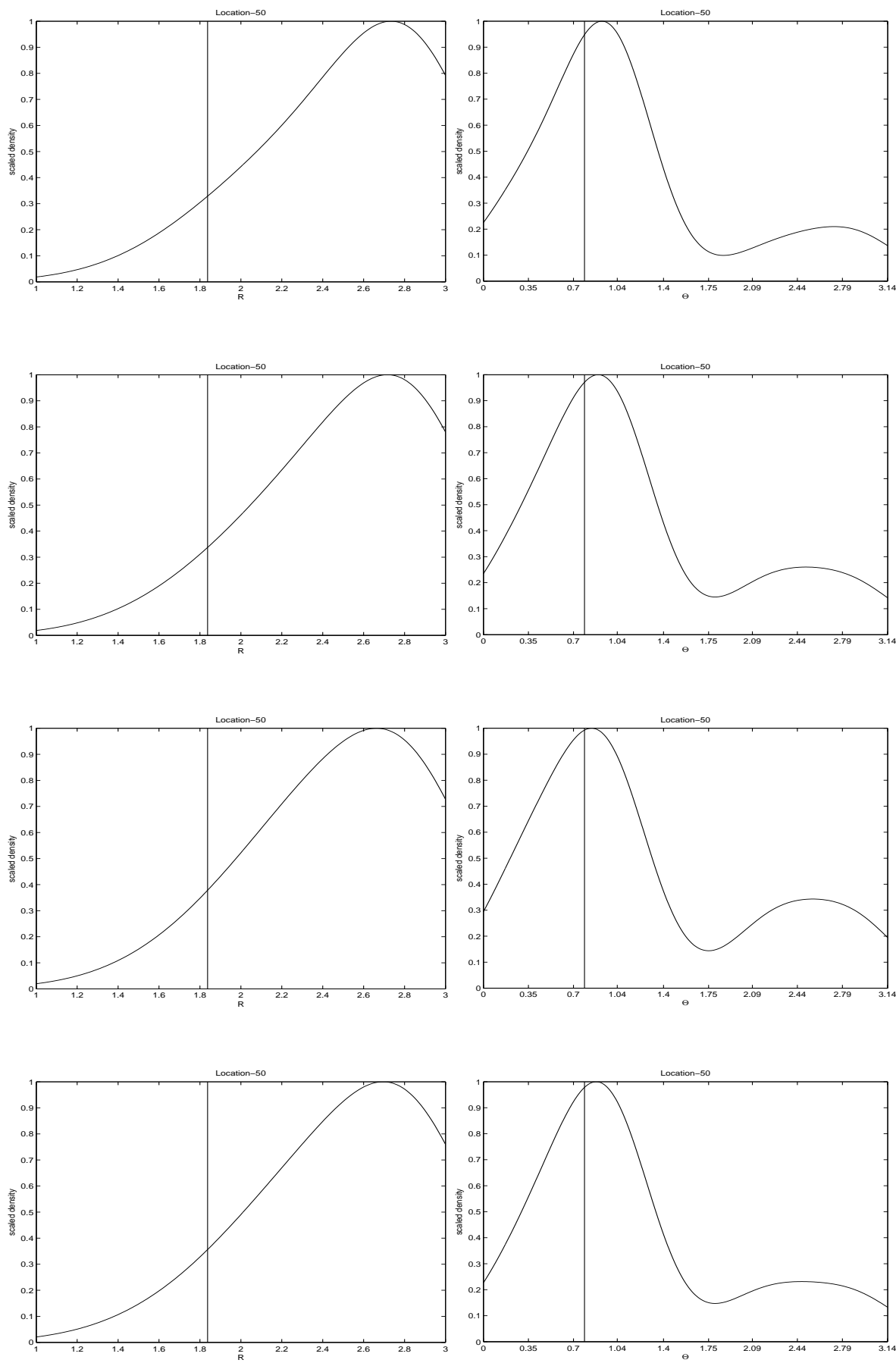

FIG 5. Real data: Leave-one-out cross-validation posteriors of the model parameter (50-th data point of the training data sets left out); the vertical line indicates the true (held out) value of $\boldsymbol{S}$. Posteriors of $R_{\odot}$ and $\Phi_{\odot}$ in the bar6 (left and right of top row of panels respectively) and sp3bar3 models (left and right of 2nd from top, respectively). Posteriors of $R_{\odot}$ and $\Phi_{\odot}$ in the sp3bar3_18 (left and right in 3rd row respectively) and sp3bar3_25 models (left and right in bottom row respectively). 
believe apriori that the true location lies in the interior of $[1.7,2.3] \times[0, \pi / 2]$, not on the boundary.

IRMCMC has an inbuilt strategy of handling multimodality by incorporating re-starts in Step b (ii) of the IRMCMC algorithm provided in Section 4.1. To clarify, given a $\left(\tilde{\boldsymbol{Q}}^{(j)}, \tilde{\boldsymbol{\Sigma}}^{(j)}\right)$, we can use independent starting points of $\boldsymbol{s}$ and a subsequent burn-in for every $j \in\left\{1, \ldots, J_{1}\right\}$, while drawing from the posterior $\left[s \mid \tilde{\boldsymbol{Q}}^{(j)}, \tilde{\boldsymbol{\Sigma}}^{(j)}, \mathcal{D}_{s}^{(-i)}, \mathbf{v}^{(t e s t, i)}\right]$. The independent initialising values can be drawn uniformly from the parameter space of $s$. This multiple re-start strategy ensures that for adequately large $J_{1}$, all the modes of the multimodal posterior are explored by IRMCMC; that is, the IRMCMC sample $\left\{\hat{\boldsymbol{s}}_{1}^{(1)}, \ldots, \hat{\boldsymbol{s}}^{\left(J_{1} J_{2}\right)}\right\}$ will then adequately represent the multimodal $i$-th cross-validation posterior (Bhattacharya and Haslett, 2007).

\subsection{Results of cross-validation on real stellar velocity data}

For our implementation of the above-discussed re-start based IRMCMC, we choose $N=20,000, J_{1}=50$, a burn-in of size 1,000 for every re-start, and $J_{2}=4,000$. Thus, IRMCMC for every cross-validation posterior yields $J_{1} J_{2}=$ $50 \times 4,000=20,000$ samples. For each of the four models, $100 \%$ observed $r_{\odot}$ fell within the $95 \%$ credible regions of their respective cross-validation posteriors. In the case of $\phi_{\odot}$, all but the minimum observed value of $\phi_{\odot}$ in the training data sets, which is about 0.08 , are captured by the respective $95 \%$ credible regions.

Figure 5 displays the cross-validation posteriors corresponding to the 50 -th data point of each of the four training data sets. The true or held out values of $R_{\odot}$ and $\Phi_{\odot}$, as inferred using our Bayesian method and TMCMC, are denoted by vertical lines in the panels of Figure 5. The cross-validation posteriors corresponding to the different models are very similar, even though the posteriors of the unknown location associated with the real test data set are quite different (recall Figures 1, 2, 3, 4 of CBB. However, there is no conflict between these two issues. Figures 1, 2, 3, 4 of CBB correspond to different training data sets, but all these have a common test data set. On the other hand, in the cross-validation scenario, while predicting a particular observed location, the held out test data sets are also different for the four different cross-validation studies. Thus for example, the test data employed in predicting the $i$-th held out data point is $\mathbf{v}^{(t e s t, i)}:=\mathbf{v}_{i}$. The cross-validation results suggest that the four different model-specific test data sets used to predict a location common to all the four models (that is, the original four training data sets) provide similar information regarding the held out location, in conjunction with the remaining model-specific training data set.

\section{Effects of chaos}

The concurrence of our results with the results reported in astrophysical literature (see Setion 4 of $\mathrm{CBB}$ ) goes beyond just the summaries of the posteriors 
of the solar position vector; high correlation is noticed between the measure of chaos in these 4 astrophysical models - as estimated by (Chakrabarty and Sideris, 2008 ) - and the multi-modality of the posterior distribution of $\boldsymbol{S}$ that we advance. Chakrabarty and Sideris (2008) report minimum chaos in the bar_6 model compared to the other three, while we notice the posteriors of both $r_{\odot}$ and $\phi_{\odot}$ in this model to be the unimodal. In fact the posteriors of $r_{\odot}$ and $\phi_{\odot}$ are unimodal only for this model, out of the 4 astrophysical models that we use to illustrate the efficacy of our method. Perhaps more importantly, the sp3bar3 model is noticed to manifest maximum (even global) chaoticity, on theoretical grounds by Chakrabarty (2007), backed by the chaos quantification at higher energies (Chakrabarty and Sideris, 2008). Likewise, in our work, the posterior distributions for $r_{\odot}$ and $\phi_{\odot}$ are most multi-modal in this model, compared to the other three. The models sp3bar $3 \_18$ and sp3bar $3 \_25$ are considered to be of intermediate chaoticity and we find these to correspond to posterior distributions (of $\boldsymbol{s}^{(n e w)}$ ) that are multi-modal, though less so, than that for the model sp3bar3.

The exact physical reason for the correlation between chaos in the base astrophysical model of the Milky Way and the multi-modality of the posterior distribution of $\boldsymbol{S}$ is understood if we begin with the premise that increased chaos is responsible for increased scatter in the distribution of the stellar velocity vector values that are generated at a chosen design vector. While for zero chaos, a distinct set of data vectors is generated at a given set of experimental conditions (a value of $\boldsymbol{S}$ ), increased scatter implies that the same data set can result from multiple experimental conditions (multiple values of $\boldsymbol{S}$ ). In fact, a necessary condition for chaos to occur is the increasing non-injectivity of $\boldsymbol{\xi}(\cdot)$ (Sengupta, 2003) where data vector $\mathbf{v}=\boldsymbol{\xi}(\boldsymbol{s})$. Thus in a base model that has zero chaoticity-eg. the bar_6 model which Chakrabarty and Sideris (2008) found to have near zero chaos-the velocity vectors generated at different values of $\boldsymbol{S}$ are distinct in general. However in the other 3 base models that were reported to bear a very high fraction of chaotic orbits, similar velocity vectors can be generated at different values of $\boldsymbol{S}$.

In summary, the function $\boldsymbol{\xi}(\cdot)$ that is learnt from the training data will be rendered increasingly more non-injective with increasing chaoticity in the base model from which the training data is generated. Thus, with increased chaoticity, $\boldsymbol{\xi}^{-1}(\cdot)$ becomes multivalued, i.e. the same observed velocity is predicted to be realised at multiple values of $\boldsymbol{S}$. The increase in the non-uniqueness of our achieved solution is thus physically motivated by the different amounts of chaos in the base astrophysical models. While this non-uniqueness can only be relieved by invoking further information-if and when such become available-our inference allows for the identification of all $s^{(n e w)}$ that are consistent with the data.

\section{References}

Bhattacharya, S. (2007). A Simulation Approach to Bayesian Emulation of Complex Dynamic Computer Models. Bayesian Analysis 2 783-816. 
Bhattacharya, S. and Haslett, J. (2007). Importance Resampling MCMC for Cross-Validation in Inverse Problems. Bayesian Analysis 2 385-408.

Binney, J. and Merrifield, M. (1998). Galactic Astronomy. Princeton University Press, Princeton.

Carlin, B. P., Polson, N. G. and Stoffer, D. S. (1992). A Monte Carlo Approach to Nonnormal and Nonlinear State-Space Modeling. Journal of the American Statistical Association 87 493-500.

Chakrabarty, D. (2007). Phase Space around the Solar Neighbourhood. Astronomy \& Astrophysics 467145.

Chakrabarty, D. and Sideris, I. (2008). Chaos in Models of the Solar Neighbourhood. Astronomy 83 Astrophysics 488161.

Dutta, S. and Bhattacharya, S. (2013). Markov Chain Monte Carlo Based on Deterministic Transformations. Statistical Methodology. To appear. Available at arxiv:1106.5850v3 with supplementary section in arxiv.org/pdf/1306.6684.

Fux, R. (2001). Order and chaos in the local disc stellar kinematics induced by the Galactic bar. Astronomy \& Astrophysics 373 511-535.

Gelfand, A. E. (1996). Model determination using sampling-based methods. In Markov Chain Monte Carlo in Practice (W. Gilks, S. Richardson and D. Spiegelhalter, eds.). Interdisciplinary Statistics 145-162. Chapman and Hall, London.

Gelfand, A. E., Dey, D. K. and Chang, H. (1992). Model determination using predictive distributions with implementation via sampling methods(with discussion). In Bayesian Statistics 4 (J. M. Bernardo, J. O. Berger, A. P. Dawid and A. F. M. Smith, eds.) 147-167. Oxford University Press.

Ghosh, A., Mukhopadhyay, S., Roy, S. and Bhattacharya, S. (2013). Bayesian Inference in Nonparametric Dynamic State-Space Models. Submitted, available at http://arxiv.org/abs/1108.3262.

Schervish, M. J. (1995). Theory of Statistics. Springer-Verlag, New York.

Sengupta, A. (2003). Toward a Theory of Chaos. International Journal of Bifurcation and Chaos 13 3147-3233. 